\title{
The form of etch rate minima in wet chemical anisotropic etching of silicon
}

\author{
M Elwenspoek $\dagger$ \\ MESA Research Institute, University of Twente, PO Box 217, NL-7500 AE \\ Enschede, The Netherlands
}

Received 23 July 1996, accepted for publication 29 August 1996

\begin{abstract}
Etching of monocrystalline silicon in alkaline based solutions leads to a deep minimum in the etch rate crystallographically oriented along $\langle 111\rangle$. The details of the form of the minimum (angular dependence of the etch rate) are investigated and discussed in a framework of steps originating from spontaneous nucleation and from misorientation of the crystal face exposed to the etchant. As a result, the etch rate minimum is characterized by a narrow flat portion that reflects the density of nuclei, and the temperature dependence of the width has an activation energy equal to $1 / 3$ of the nucleation barrier.
\end{abstract}

This article deals with the dependence of the silicon etch rate close to the $\langle 111\rangle$ orientation when etching in anisotropic etching solutions such as aqueous $\mathrm{KOH}$ and EDP $\ddagger$. In these solutions the etch rate is strongly dependent on the crystallographic orientation of the silicon single crystal, with absolute minima in $\langle 111\rangle$, and relative minima in $\langle 001\rangle$ (aqueous $\mathrm{KOH}$ ) and $\langle 110\rangle$ (aqueous $\mathrm{KOH}$ saturated with isopropyl alcohol, IPA and EDP). An extensive discussion on experimental results can be found e.g. in [1]. The origin of the anisotropy is being debated in the literature. While many authors try to find explanations based on (electro-) chemical reactions of water or hydroxyl ions with the silicon surface for orientation dependent contributions [1-4], this author proposed to use models developed for the rate of crystal growth to find the origin of the anisotropy [5-7]. This view has consequences on some details of the etch rate diagram (i.e. the variation of the etch rate with the crystallographic orientation) that will be explored here and compared to experimental data taken from the literature. In our view, understanding the anisotropy of the etch rate of silicon (and other singlecrystalline materials, such as quartz and GaAs) is not only interesting from a scientific point of view, but also for the development of micromachining. Wet chemical etching is a key technology for micromachining because it allows fast, precise and reproducible shaping of mechanical microcomponents such as numerous sensors for pressure, force, acceleration, flow, temperature and concentration of chemicals, actuators like pumps, valves, switches and microstructures such as microsieves, canals and nozzles. The major volume is currently being fabricated in research

$\dagger$ email: m.elwenspoek@eltn.utwente.nl

$\ddagger$ Ethylenediamine, pyrocathecol and water mixtures, occasionally with traces of pyridine. laboratories, but scaling up to mass production makes perfect control of the technology necessary, which is very difficult to achieve if the origin of a prominent and important property of the etching - the anisotropy-is not understood. This is a quite formidable task, and this paper will contribute a step towards executing to it.

Starting with a short outline of the crystal growth view on wet chemical anisotropic etching, we focus on the kinetics of steps on misoriented flat crystal faces. We have found that there are two competing mechanisms for the production of steps on crystal faces of perfect crystals (spontaneous formation of steps by two-dimensional nucleation $\S$ and the steps due to misorientation). The peculiar interaction of steps leads to a picture in which the more effective step source dominates the etch rate, resulting in a very interesting shape of the minimum. This shape has been found by Uenishi et al [8] for $\mathrm{Si}\langle 111\rangle$. Earlier results [1,9] agree with Uenishi's results but due to a much smaller angular resolution, this particular shape was not apparent. Similar results, but less pronounced, are reported for $\mathrm{Si}\langle 001\rangle$ [6].

It is well established that the growth form of crystals is dominated by a small number of flat faces on crystals. The flat face is synonymous with a cusp in the surface free energy and a finite step free energy. The latter tells us that one has to work in order to create a step on a flat surface. If this work were equal to zero, the surface would spontaneously be rough, maximizing this way the entropy of the system. It means also that there is a twodimensional barrier to create a step, e.g., in the form of a circular cavity of radius $R$. This is quite analogous to classical nucleation theory. It is simply a consequence of a

$\S$ Nucleation in this paper refers to nucleation of etchpits. 


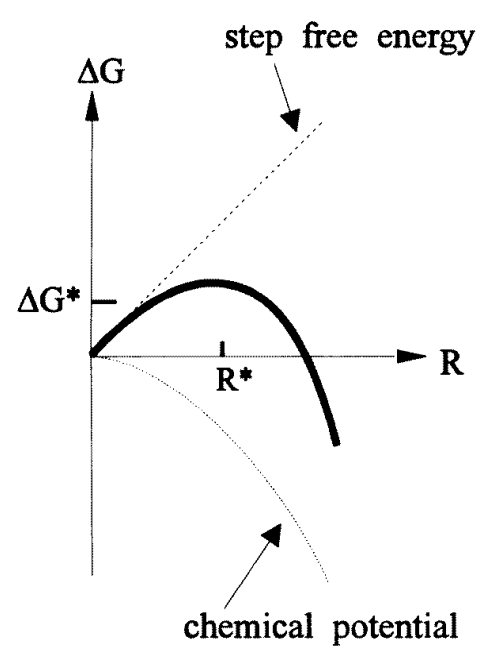

Figure 1. The free energies involved in the formation of an etchpit of radius $R$. Free energy is gained by etching, which leads to a negative contribution proportional to the number of atoms removed from the pit, which is in turn proportional to the area of the pit, leading to a parabolic dependence of the free energy on the pit radius. The formation of a step associated with pit formation costs energy, its contribution is positive, and it is proportional to the length of the circumference of the pit, and hence proportional to its radius. The sum is the total energy change, and exhibits a maximum at $R^{*}$ with a height $\Delta G^{*}$. This maximum constitutes the classical nucleation barrier.

competition of a free energy reduction by dissolving atoms (this free energy equals the driving force) and an increase of the surface area by removing atoms from a flat face by making steps. To create a cavity (the nucleus), one has to remove atoms from the surface and one has to create a step; the first contribution is proportional to $R^{2}$ and negative (for etching and dissolving) and the latter is proportional to $R$ and positive, see figure 1 . We get a maximum if we plot the reversible work to be done as a function of $R$, at

$$
R^{*}=b \Delta \mu / \gamma
$$

with a height

$$
\Delta G^{*}=\gamma^{2} / \Delta \mu .
$$

$\gamma$ is the free energy of an atom in a step, $b$ is the length of the atom in the step and $\Delta \mu$ is the undersaturation, the driving force. We ignored factors of the order unity that arise from the shape of the nucleus in both formulae. A somewhat more complete discussion for the case of silicon etching can be found in [5], and a general discussion on the critical nucleus is given by van Leeuwen and Bennema [10]. The etch rate of a perfectly aligned flat face of a perfect crystal (i.e. without dislocations) will contain a term (see e.g. [11])

$$
E \propto \exp -\Delta G^{*} / k T=\exp -\gamma^{2} / k T \Delta \mu .
$$

The other factors that determine the etch rate will be the speed of moving steps and the concentration of etching species at the crystal surface. The former will depend on the chemical reaction rate of the fastest step in a reaction sequence $\tau^{*-1}$ and the surface diffusion, the latter on concentration in the etching solution and volume transport $\dagger$.

For etching silicon, $\Delta \mu$ must be very large ( $3 \mathrm{eV}$ is the energy of oxidation of silicon) $\ddagger$. If nucleation has any serious influence on the etch rate, $\Delta G^{*} / k T$ must be of the order of one or larger and the size of the critical nucleus must obviously be larger than one atom. This means that $\gamma$ must also be quite large, if $\Delta \mu$ is this large. Since the difference in the activation energy of $\langle 111\rangle$ and the other faces is of the order of $0.1 \mathrm{eV}$, we might expect that $\Delta G^{*}$ is close to $0.1 \mathrm{eV}$. This is also evidenced by the fact that the activation energy of the velocity of steps on $\langle 111\rangle$ etched in $\mathrm{KOH}$ is $0.6 \mathrm{eV}$, again $0.1 \mathrm{eV}$ smaller than the activation energy of the $\langle 111\rangle$ etch rate [7]. $\Delta \mu=3 \mathrm{eV}$ and $\Delta G^{*}=0.1 \mathrm{eV}$ conflicts with the requirement of having a critical nucleus of at least one atom.

Flat faces can often be found from the crystallography, however, in the diamond lattice, crystallography provides us with only the $\langle 111\rangle$ face as a flat face. Other faces can be flat due to additional surface effects such as adsorption and surface reconstruction. We ignore these effects here, because we concentrate on the $\langle 111\rangle$ which does not seem to be reconstructed, at least when etching in $\mathrm{NaOH}$ at room temperature [12].

In the paper by Allongue et al [12] there are fine STM images which show moving steps on atomically flat $\langle 111\rangle$ silicon while etching in $\mathrm{NaOH}$ solutions.

Let us turn now to the etching of misoriented $\mathrm{Si}\langle 111\rangle$ faces. In figure 2 we give a schematic of a flat face with steps on it. These steps are due to a misorientation which is imposed by the experiment. The etching proceeds by movement of the steps of height $h$, and the average distance between the steps is $\lambda$. The speed of the steps $v$ is given by

$$
v=\lambda / \tau
$$

where $\tau$ is the time a step needs to move the distance $\lambda$. After time $\tau$ the crystal surface has moved a distance $D$, and the etch rate $E$ is given by

$$
E=D / \tau \text {. }
$$

Elementary geometry now gives the following expression of the etch rate:

$$
E=v \sin \Theta \approx \nu \Theta .
$$

$\dagger$ The effect of doping e.g. by B to reduce the etch rate is isotropic, and it must be modelled in the chemical reaction rate $\tau^{*-1}$. The influence of a bias on the etch rate is subject to further investigation. A bias may change the bond energy and the chemical potential difference, and it might also change the step free energy. Anodic oxidation is not included in this picture; it seems that at the passivation potential the character of the electrochemical reaction changes. The nature of the etching medium affects in principle all parameters. It is worth noting that under certain conditions there appears a minimum of the etch rate in $\langle 110\rangle$, but there are only a few data that show both minima in $\langle 001\rangle$ and $\langle 110\rangle$ simultaneously. There seems to be a systematic trend: if large molecules are present in the solution, the minimum in $\langle 110\rangle$ becomes more pronounced (in EDP and when adding IPA to the solution). Perhaps these molecules bridge the parallel running bond chains on the $\langle 110\rangle$ oriented silicon face, stabilizing the face and resulting in a non-zero step free energy on $\langle 110\rangle$.

$\ddagger$ Care must be taken since it is the chemical potential of the chemical species that leaves the surface, and this is not necessarily $\mathrm{Si}(\mathrm{OH})_{4}$. 


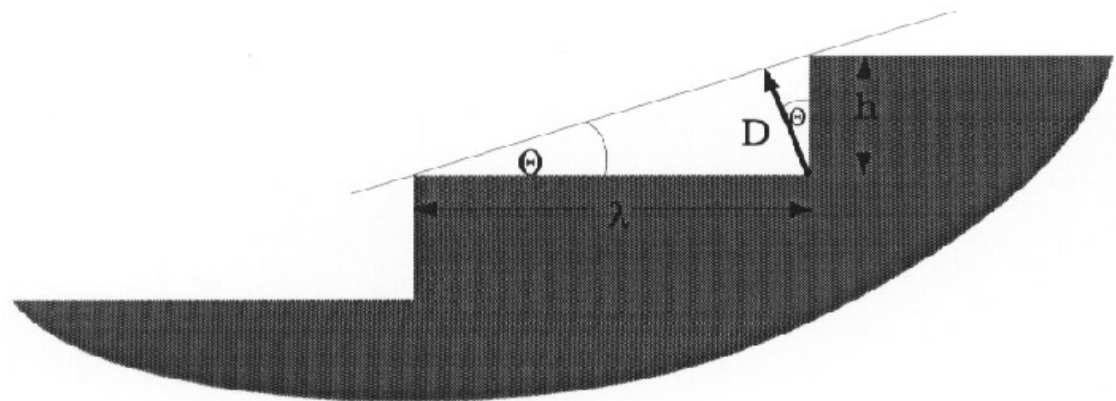

Figure 2. Illustration for the derivation of the relations between step height $h$, distance between steps $\lambda$, angle of misorientation $\Theta$ and the projection of misoriented crystal front $D$, when the step has moved the distance $\lambda$.

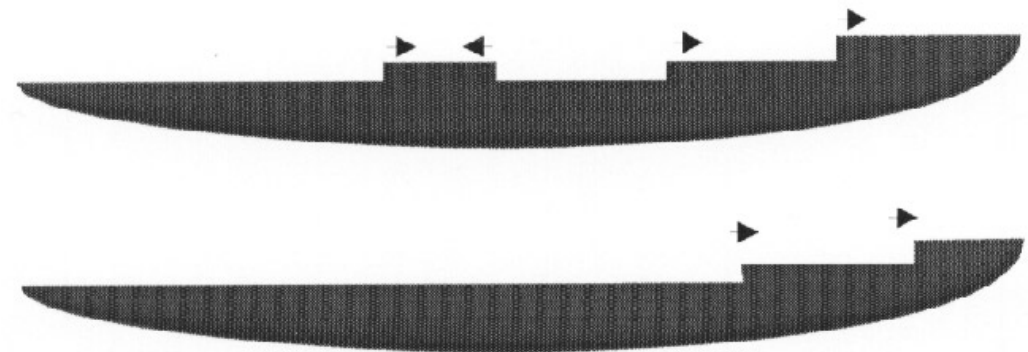

Figure 3. Annihilation of steps by collision.

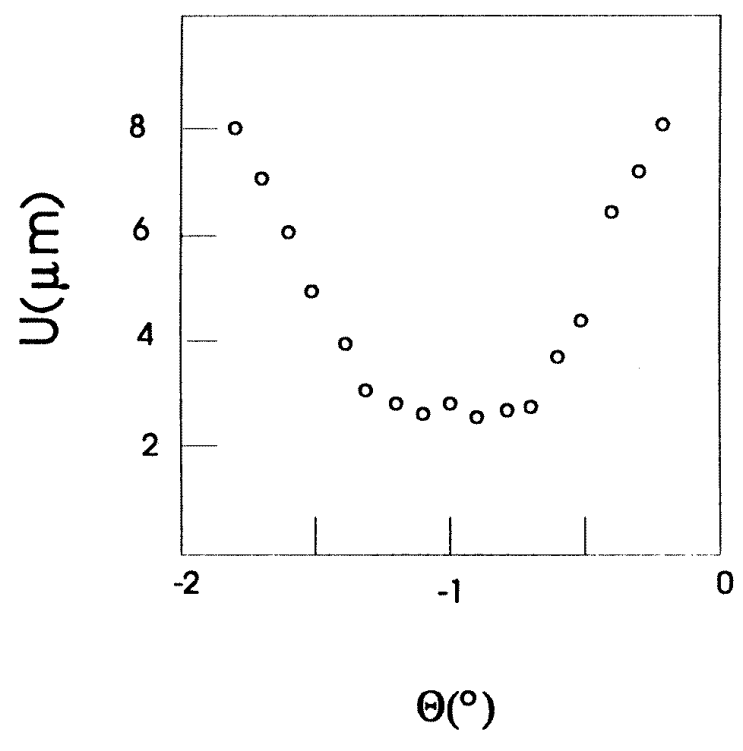

Figure 4. Underetching close to the $\langle 111\rangle$ orientation of $\langle 110\rangle$ oriented silicon wafers in $40 \mathrm{wt} \% \mathrm{KOH}$ at $70^{\circ} \mathrm{C}$ (redrawn from Uenishi et al [8]). The etch depth was $350 \mu \mathrm{m} . \Theta=0^{\circ}$ refers to the orientation of the flat.

The last equality applies for a small misalignment.

From (6) we see that the velocity of the steps is given by the derivative of the etch rate with respect to the angle of misorientation close to $\langle 111\rangle$. In the temperature dependence there is no nucleation barrier. The velocity of steps is only given by the chemical reaction rate and transport along the surface and in the volume. The difference of the activation energy of the $\mathrm{Si}\langle 111\rangle$ etch rate and the activation energy of the velocity steps yields the height of the nucleation barrier $\Delta G^{*}$. Using the data of Koide et al [13] we found in [7] an activation energy for the step velocity on $\mathrm{Si}\langle 111\rangle$ of $0.6 \mathrm{eV}$, which must be compared with the $\mathrm{Si}\langle 111\rangle$ etch rate having an activation energy of $0.7 \mathrm{eV}[1]$.

This dependence changes if the distance between the steps becomes wide, i.e. if $\Theta$ becomes very small. Eventually the density of nuclei between the steps becomes so large that the total length of steps due to the misorientation becomes smaller than the length of the steps due to nucleation.

This situation is shown in figure 3 . In the upper part a nucleus exists between the steps. The two steps on the left collide by their motion and annihilate each other. The presence of the nucleus changes the number of steps only for a short time: we can say that their steps are annihilated by the steps originating from the misorientation. If there were more nuclei, 'their steps' would annihilate the 'misorientation steps'. The interplay of the steps originating from the two step sources would almost work like a switch: the etch rate is given by the step mechanism that provides the larger number of steps.

The nucleation rate will be independent of the orientation; close to $\langle 111\rangle$ the etch rate does not depend on the orientation. When $\Theta$ becomes large enough, the misorientation steps take over and the etch rate varies as $\sin \Theta$.

This angular dependence of the etch rate is observed by Uenishi et al [8]: a small portion of the curve is 
independent of the orientation, and there is a steep transition to a linear dependence of the etch rate on $\Theta$. The experimental results are redrawn in figure 4.

We will have the turnover at an angle $\Theta_{c}$ corresponding roughly to a step distance $\lambda_{c}$ if the nucleation rate $J$, which is the number of cavities per unit time and surface, divided by $\lambda_{c}^{2}$ and $\tau$ equals unity,

$$
J / \lambda_{c}^{2} \tau=1
$$

We have $\lambda / \tau=v \approx d / \tau^{*}$, with $d$ the width of an atom in a step and $\tau^{*} \propto 1 / \kappa$, the chemical reaction rate to remove a single atom from the step. $\lambda_{c}$ is related to the misorientation by

$$
\lambda_{c}=h / \tan \Theta_{c} \approx h / \Theta_{c} .
$$

The nucleation rate at very large undersaturation is given by [11]

$$
J \propto \tau^{*-1} \exp -\gamma^{2} / k T \Delta \mu
$$

and we find using (7)-(9)

$$
\Theta_{c} \propto \exp \left\{-\gamma^{2} / 3 k T \Delta \mu\right\}
$$

for the temperature dependence of the flat portion in the $E$ versus $\Theta$ plot close to the orientation of a flat face.

This is an interesting result. The temperature dependence of the width of the flat portion should be much smaller than the temperature dependence of the etch rate itself: the chemical rate $\tau^{*-1}$ drops out, and the temperature dependence is only given by the energy of the nucleation barrier divided by three. An experimental determination of the temperature variation of $\Theta_{c}$ directly gives the free energy of the critical nucleus. Unfortunately, the temperature dependence of $\Theta_{c}$ is not published in the literature, but $\Theta_{c}$ has been determined at least at one temperature by Uenishi et al. In figure 4 we have redrawn his results.

We find $\Theta_{c}=0.6^{\circ}=0.01 \mathrm{rad}$, meaning that terraces 100 times wider than their height should show many nuclei.

Our own experiments on the etch rate close to $\langle 001\rangle$ [6] revealed quite a small temperature dependence of $\Theta_{c}$ for the $\langle 001\rangle$ orientation. In figure 5 we show an Arrhenius plot of $\Theta_{c\langle 001\rangle}$ using the data from [6]. The activation energy is as small as $100-200 \mathrm{~K}(0.01 \mathrm{eV}$, much smaller than the activation energy of the $\langle 001\rangle \mathrm{Si}$ etch rate itself) indicating a very small step free energy as expected for the low minimum in the etch rate with orientation.

If the estimate of $\Delta G^{*}=0.1 \mathrm{eV}$ is correct, we expect an activation energy of $\Theta_{c<111\rangle}$ of only $0.03 \mathrm{eV}$. This should be tested experimentally.

Further we can conclude from the comparison of the activation energy for $\Theta_{c\langle 001\rangle} \approx 0.01 \mathrm{eV}$ that $\gamma_{\langle 111\rangle}=$ $10 \gamma_{\langle 001\rangle}$, in agreement with the great anisotropy of the etch rate of silicon in $\mathrm{KOH}$.

We see that the approach using the notion of steps and nucleation is quite powerful in understanding anisotropic etching of silicon. However, the problem of expecting a large driving force of at least $3 \mathrm{eV}$, in conflict with a small free energy of the critical nucleus, deserves further research. If $\Delta \mu=3 \mathrm{eV}$, the classical nucleation picture is wrong and must at least be modified. If the nucleation

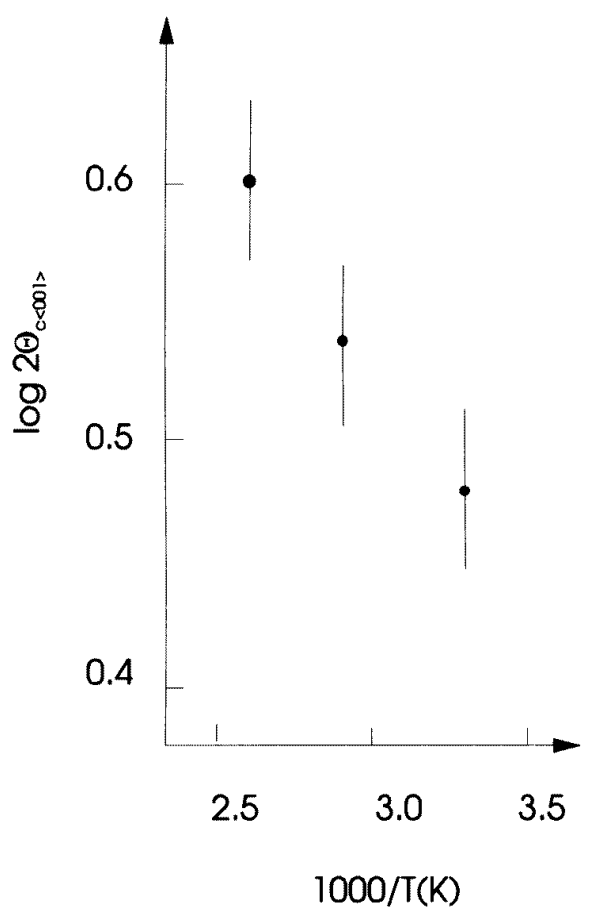

Figure 5. Temperature dependence $\Theta_{c}$ for etching $\langle 001\rangle$ silicon in aqueous $\mathrm{KOH}(40 \mathrm{~g} / 100 \mathrm{ml})$.

picture is correct, the driving force must be much smaller. A discussion on the driving force in silicon wet chemical etching will be published elsewhere.

In conclusion, we have explained with this simple picture the peculiar angular variation of the silicon etch rate close to the $\langle 111\rangle$ orientation by interaction of steps originating from competing sources: misorientation and spontaneous nucleation. Analysis shows that the temperature dependence of the width of the flat portion of the etch rate versus orientation gives directly the activation energy for nucleation, which is, in the simple two-dimensional nucleation picture, equal to the energy of the critical nucleus.

We expect a sharp increase of the activation energy in the transition regime from misoriented dominated steps (etch rate proportional to the angle) and the regime where the steps mainly originate from nucleation (flat portion). The angular variation set great demands on the wafer orientation for the determination of the true $\langle 111\rangle$ etch rate and its temperature dependence.

\section{Acknowledgment}

The author acknowledges Jaap van Suchtelen (University of Nijmegen) for helpful discussions.

\section{References}

[1] Seidel H, Csepregi L, Heuberger A and Baumgärtel H 1990 Anisotropic etching of crystalline silicon in alkaline solutions J. Electrochem. Soc. 137 3612-26 
[2] Allongue P, Kosta-Kieling V and Gerischer H 1993 Etching of silicon in $\mathrm{NaOH}$ solutions II. Electrochemical studies on $\mathrm{n}-\mathrm{Si}(111)$ and (100) and mechanisms of the dissolution J. Electrochem. Soc. 140 1018-26

[3] Glembocki O J, Stahlbush R E and Tomkiewicz M 1985 Bias dependent etching of silicon in aqueous $\mathrm{KOH} J$. Electrochem. Soc. 132 145-51

[4] Bressers P M M C, Pagano S A S P and Kelly J J 1995 Ferricyanide reduction as a probe for the surface chemistry of silicon in aqueous alkaline solutions $J$. Electroanal. Chem. 391 159-68

[5] Elwenspoek M 1993 On the mechanism of anisotropic etching of silicon J. Electrochem. Soc. 1402075

[6] Elwenspoek M, Lindberg U, Kok H and Smith L 1994 Wet chemical etching mechanism of silicon Proc. Micromechanical Systems (MEMS) (Oiso, 1994) pp 223-8

[7] Elwenspoek M 1995 Physical chemistry of wet chemical anisotropic etching of silicon Proc. ASME Dynamic Systems and Control Division vol 2, DSC-Vol 57-1 pp 901-8
[8] Uenishi Y, Tsugai M and Mehregany M 1995 Micro-opto-mechanical devices fabricated by anisotropic etching of (110) silicon J. Micromech. Microeng. 5 $305-12$

[9] Kendall D L 1975 On etching very narrow grooves in silicon Appl. Phys. Lett. 26 195-8

[10] van Leeuwen C and Bennema P 1975 The critical nucleus Surf. Sci. 51 109-30

[11] Gilmer G H 1977 Computer simulation of crystal growth $J$. Crystal Growth 42 3-10

[12] Allongue P, Kosta-Kieling V and Gerischer H 1993 Etching of silicon in $\mathrm{NaOH}$ solutions I. In situ scanning tunnelling microscopic investigation of $\mathrm{n}-\mathrm{Si}(111) \mathrm{J}$. Electrochem. Soc. 140 1009-18

[13] Koide A, Sato K and Tanaka S 1991 Simulation of two-dimensional etch profile of silicon during orientation dependent anisotropic etching Proc.

Microelectromechanical Systems (MEMS) (Nara, 1991) pp 216-20 